\title{
CORRECTION
}

\section{Statin therapy for skeletal dysplasia}

\section{Jennifer Sargent}

Nat. Rev. Endocrinol. advance online publication 30 September 2014; doi:10.1038/nrendo.2014.173

In the version of this article originally published online and in print, the doi for the original article was cited incorrectly. The doi should have read $10.1038 /$ nature 13775 not

$10.1038 /$ nature13665. This has now been corrected online. 